\title{
P02-1-22 Poster session
}

\section{Inhibition of HDAC3 preconditions the brain against ischemic}

\author{
Linyin Feng, Xiaoyu Yang, Lei Zhang
}

\section{Dept. of Neuropharmacology, Shanghai Institute of Materia Medica, Chinese Academy of Sciences, China}

Brain ischemia, induced by transient or permanent interruption of blood supply, is the major cause of mortality and morbidity worldwide. Despite extensive efforts, effective treatments for brain damage remain the unfilled medical needs in clinical investigation. Ischemic preconditioning (IPC) is an approach in which a sub-lethal ischemic exposure evokes protection against a subsequent, more severe ischemic insult. Not only a therapy applied in individuals, induced ischemic tolerance is also an attractive strategy to obtain insights into endogenous protective mechanisms. Different genomic profiles and epigenetic reprogramming of the brain have been reported between ischemic preconditioning and ischemic injury. However, less is known about the specific role of HDAC in this process.

To address this notion, PC and transient middle cerebral artery occlusion (MCAO) were conducted in Sprague-Dawley rats. Additionally, primary cultured cortical neurons were used to identify the modulators and effectors of HDAC3 involved in PC. We found that the acetylation level of histone was up-regulated, and consistently nuclear localization of HDAC3 was significantly reduced following PC in vivo and in vitro. Treatment with the HDAC3-specific inhibitor, RGFP966, mimicked the neuroprotective effects of PC $24 \mathrm{~h}$ and $7 \mathrm{~d}$ after MCAO, causing a reduced infarct volume and less Fluoro-Jade $\mathrm{C}$ staining. Improved functional outcomes were observed in the neurological score and rotarod test. We further showed that attenuated recruitment of HDAC3 to promoter regions following PC potentiates transcriptional initiation of genes including Hspa1a, Bcl211, and Prdx2, which may underlie the mechanism of protection. In addition, PC-activated calpains were implicated in the cleavage of HDAC3. Pretreatment with calpeptin blockaded the attenuated nuclear distribution of HDAC3 and the protective effect of $\mathrm{PC}$ in vivo.

Collectively, these results demonstrate that the inhibition of HDAC3 preconditions the brain against ischemic insults, indicating a new approach to evoke endogenous protection against stroke. 